Harry J Pick ${ }^{1 *}$, Mark A Faghy ${ }^{2 *}$, Gareth Creswell ${ }^{3}$, Deborah Ashton ${ }^{1}$, Charlotte E Bolton ${ }^{1}$, Tricia McKeever', Wei Shen Lim', Thomas Bewick ${ }^{3}$

'Nottingham University Hospital NHS Trust, Nottingham City Hospital, NG5 1PB Nottingham, United Kingdom

${ }^{2}$ University of Derby, Kedleston Road, DE22 1GB Derby, United Kingdom

${ }^{3}$ University Hospitals of Derby and Burton NHS Foundation Trust, Uttoxeter Road, DE22 3NE Derby, United Kingdom

${ }^{*}$ Authors contributed equally to this work.

\title{
The feasibility and tolerability of using inspiratory muscle training with adults discharged from the hospital with community-acquired pneumonia
}

\begin{abstract}
Introduction: Patients experience substantial morbidity following discharge from hospital and during recovery from community-acquired pneumonia (CAP). Inspiratory muscle training (IMT) has demonstrated improved functional capacity and reduced patient-reported symptoms. To date the safety and tolerability of these methods have not been determined in CAP patients recovering following hospitalization. Accordingly, this study aimed to assess the safety and tolerability of IMT in adults discharged from hospital with CAP.

Material and methods: Participants received an IMT device (POWERbreathe KHP2) and completed 9-weeks IMT training with weekly follow-up. Frequency (twice daily) and load (50\% PImax) were fixed throughout, but training volume increased incrementally (2-week habituation phase, 7-week training phase). Primary outcomes of interest included IMT safety and tolerability. Results: Twenty-two participants were recruited; 16 were male, mean age 55.2 years (range 27.9-77.3). From 1183 possible training days, side effects were reported on 15 occasions by 10 individual participants. All reported side-effects were assessed as grade 1 and did not prevent further training. Participant-reported IMT acceptability was $99.4 \%$.

Conclusion: Inspiratory muscle training is safe and tolerable in patients following hospitalisation for CAP. Patient satisfaction with IMT is high and it is viewed by patients as being helpful in their recovery. Distinguishing CAP-related symptoms and device-related side effects is challenging. Symptom prevalence declined during follow-up with concurrent improvements in spirometry observed. Further research is required to determine the efficacy of IMT interventions following CAP and other acute respiratory infections.
\end{abstract}

Key words: recovery; pnuemonia; respiratory muscles; training

Adv Respir Med. 2021; 89: 216-220

\section{Introduction}

CAP is responsible for over 100,000 admissions to hospital each year in England and Wales with an incidence of 8 per 1000 adults aged over $65[1,2]$. Most hospitalised patients are discharged into the community to recuperate and the burden of disease in recovery is substantial, with $65.7 \%$ of patients re-consulting a primary care practitioner, and $>50 \%$ of patients failing to return to their usual daily activities in the 4-weeks post-discharge [3]. Whilst the burden of recovery is extensive the physiological basis for recovery remains poorly understood. Previous work in this area has demonstrated reduced skeletal muscle strength, impaired exercise capacity and reduced quality of life following an episode of CAP [4]. A potential mechanism is abnormal and impaired respiratory muscle function in the post-pneumonia period. In healthy volunteers, respiratory muscle strength is associated with exacerbated dyspnoea and fatigue, heightened perceptual discomfort and reduced exercise capacity and functional status. During ventilation, 
breathing accounts for 1-3\% of total oxygen consumption and quiet breathing requires a small fraction of the respiratory muscles maximal pressure generating capacity in healthy individuals. However, in an acutely diseased lung (i.e. CAP) the pressure required to breathe is increased due to changes in airway resistance and chest wall mechanics [5].

Inspiratory muscle training (IMT) improves respiratory muscle strength and reduces markers of physiological stress [6]. IMT techniques have also been demonstrated in patient groups; reducing dyspnoea perception and discomfort in chronic obstructive pulmonary disease, increased strength and endurance of respiratory muscles in patients after a cerebrovascular event and increased lung volumes, respiratory muscle strength, and exercise capacity in elderly patients $[7,8]$. The efficacy and tolerability of IMT in CAP patients have yet to be determined.

\section{Material and methods}

We undertook a prospective observational feasibility study with adult patients discharged from hospital with CAP. CAP was defined as: the presence of one or more symptom of acute lower-respiratory respiratory-tract infection (cough, increasing breathlessness, sputum production, chest pain, and fever); evidence of acute infiltrates consistent with a respiratory infection on admission plain chest radiograph; , and treated by the clinical team for CAP. Exclusion criteria included; inability to understand verbal or written information in English, hospital admission within preceding 10 days, active tuberculosis, post-obstructive pneumonia secondary to lung cancer, aspiration pneumonia, World Health Organisation performance status 2 or greater prior to admission, inability to provide informed consent, or presence of a contraindication to pulmonary function testing.

Following ethics approval (Ethics reference 17/EE/0043) and informed consent, twenty-two adults (average age was 55.2 years; range: 27.977.3 years, mean CAP score $44.3 \pm 19.6$ ) and $16(72.7 \%)$ males were recruited to the study and completed 9 weeks of IMT training (described below). One or more co-morbid illness was present in 10 patients. World Health Organisation (WHO) performance status at hospital admission was grade 1 in nine, grade 2 in three, grade 3 in seven, and grade 4 in three patients. A control group was not included within the study design as the primary aim was to determine the safety and tolerability of IMT methods in patients following hospitalisation for CAP. Most patients (21/22) were admitted from the Emergency Department, with three patients taking prescribed antibiotics before admission. Unilobar consolidation was the most common radiographic abnormality identified following admission (17/22 patients), followed by multilobar consolidation $(4 / 22)$ and unilateral pleural effusion (1/22).

Following informed consent and alongside routine clinical investigations, patients were assessed for symptom prevalence and functional activity weekly for 9 weeks using a combination of hospital review, telephone consultation, and patient diaries. A five-item, CAP specific questionnaire was used to record patient-reported symptoms prior to discharge and at each follow-up consultation, scoring was conducted inline with previous guidelines [9]. Dynamic spirometry (forced expiratory volume in 1 second; $\mathrm{FEV}_{1}$ and Forced Vital Capacity; FVC) was assessed using a handheld spirometer (MicroPlus; Micro Medical, Buckinghamshire, UK) in accordance with published guidelines [10]. A hand-held mouth pressure meter (Micro R.P.M., Micro Medical, Buckinghamshire, UK) was used to assess both maximal inspiratory (MIP) and maximal expiratory (MEP) mouth pressure. Patients were provided with an IMT device (Figure 1, POWERbreatheKHP2, HaB International, Southam, UK) for nine weeks. Patients conducted two IMT sessions at home each day an intensity equivalent to $50 \%$ of the participant's maximum inspiratory muscle pressure (MIP). Participants were trained on using the device and completing sessions effectively

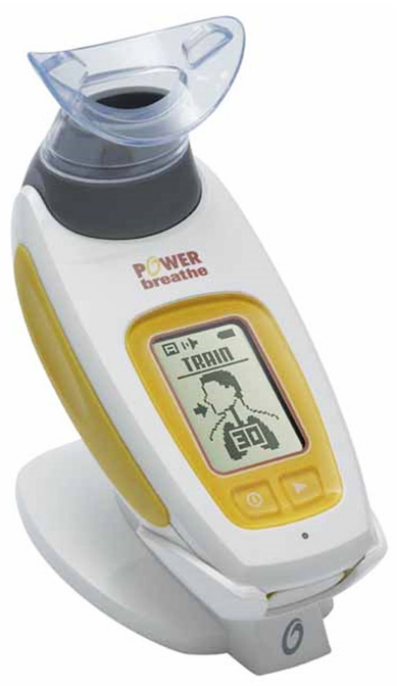

Figure 1. A visual representation of the POWERbreatheKHP2 device that was used by patients as part of the 9 -week intervention 
CAP. This study demonstrates that IMT in adult patients recovering following hospitalisation with CAP is safe and tolerable. We also observed improvement in CAP-symptom scores and lung function tests as would be expected in recovery. The authors acknowledge that the efficacy of IMT methods cannot be determined by this study and the full extent to which IMT may have influenced the rate or extent of improvement in recovery due to the lack of a control group and should be considered with future research. Specifically, the use of IMT methods needs to be evaluated against the low-grade side-effects experienced by patients and the protocol adherence to determine the full extent of the benefits to patients.

We do present the first study that demonstrates that IMT methods are safe and well tolerated by patients following admission to hospital with CAP. Reported side-effects were rare and of low severity, rated as grade 1 on Common Terminology Criteria for Adverse Events scale (CTCAE), and did not prevent further training or deviation from the study protocol. This is consistent with results from a study of IMT in patients with thoracic malignancy which reported IMT-related side effects in only 3 patients during follow-up whilst 2 studies of IMT in differing patient cohorts (pre-operative IMT in patients undergoing coronary artery bypass grafting and IMT in chronic heart failure) both reported no adverse events during or after IMT. On repeated assessment patients reported that they believed IMT was helpful in their recovery. This is consistent with results of IMT from other studies; pre-operative patients and patients with chronic heart failure participating in rehabilitation also reported that IMT was tolerable and that they believed it to be beneficial in their recovery [11] FEV1, $24+/-7 \%$ predicted.

It is plausible that IMT methods could also have important implications for patients during their recovery from COVID-19 [12]. COVID-19 and other viral infections can cause significant damage to the lungs and airways result in acute respiratory distress syndrome (ARDS). The patients at the greatest risk are likely to have multiple co-morbidities, de-conditioning of the respiratory musculature and increased likelihood of respiratory failure and the need for critical care interventions. Those that develop severe complications from are admitted to intensive care units and require prolonged periods of ventilation. Mechanical ventilation induces rapid atrophy and profound weakness of the respiratory musculature ( $<18$ hours), creating a disparity between the force-generating capability of the respiratory muscles and the pressures required for spontaneous tidal breathing [13]. Interventions that increase respiratory muscle strength are well tolerated by patients with respiratory illness and the data here demonstrates that the inclusion of IMT techniques could prove a useful addition to the recovery from respiratory infections.

\section{Acknowledgements}

The authors wish to thank the clinicians and staff of Nottingham University Hospitals NHS Trust and University Hospitals of Derby and Burton NHS Foundation Trust without whom the study would not have been possible; the Nottingham Pneumonia Patient and Public Involvement group for their significant ongoing input to this project and the additional research studies of the respiratory infection research team; and all participants who gave their consent for this study.

\section{Conflict of interest}

None declared.

\section{References:}

1. Chalmers J, Campling J, Ellsbury G, et al. Community-acquired pneumonia in the United Kingdom: a call to action. Pneumonia (Nathan). 2017; 9: 15, doi: 10.1186/s41479-017-0039-9, indexed in Pubmed: 29043150.

2. Trotter CL, Stuart JM, George R, et al. Increasing hospital admissions for pneumonia, England. Emerg Infect Dis. 2008; 14(5): 727-733, doi: 10.3201/eid1405.071011, indexed in Pubmed: 18439353.

3. Pick HJ, Bolton CE, Lim WS, et al. Patient-reported outcome measures in the recovery of adults hospitalised with community-acquired pneumonia: a systematic review. Eur Respir J. 2019; 53(3), doi: 10.1183/13993003.02165-2018, indexed in Pubmed: $\underline{30635298 .}$.

4. José A, Corso SD. Patients hospitalized for community-acquired pneumonia present reduced functional performance. Braz J Phys Ther. 2013; 17(4): 351-358, doi: 10.1590/S141335552013005000098, indexed in Pubmed: 24072224.

5. Levine S, Nguyen T, Taylor N, et al. Rapid disuse atrophy of diaphragm fibers in mechanically ventilated humans. N Engl J Med. 2008; 358(13): 1327-1335, doi: 10.1056/NEJMoa070447, indexed in Pubmed: 18367735.

6. Illi SK, Held U, Frank I, et al. Effect of respiratory muscle training on exercise performance in healthy individuals: a systematic review and meta-analysis. Sports Med. 2012; 42(8): $707-$ 724, doi: 10.1007/BF03262290, indexed in Pubmed: 22765281.

7. Beaumont M, Forget P, Couturaud F, et al. Effects of inspiratory muscle training in COPD patients: A systematic review and meta-analysis. Clin Respir J. 2018; 12(7): 2178-2188, doi: 10.1111/crj.12905, indexed in Pubmed: 29665262.

8. Charususin N, Gosselink R, Decramer M, et al. Randomised controlled trial of adjunctive inspiratory muscle training for patients with COPD. Thorax. 2018; 73(10): 942-950, doi: 10.1136/thoraxjnl-2017-211417, indexed in Pubmed: 29914940.

9. El Moussaoui R, Opmeer BC, Bossuyt PMM, et al. Development and validation of a short questionnaire in community acquired pneumonia. Thorax. 2004; 59(7): 591-595, doi: 10.1136/thx.2003.015107, indexed in Pubmed: 15223867. 
10. American Thoracic Society/European Respiratory Society. ATS/ERS Statement on respiratory muscle testing. Am J Respir Crit Care Med. 2002; 166(4): 518-624, doi: 10.1164 rccm.166.4.518, indexed in Pubmed: 12186831.

11. Ramirez-Sarmiento A, Orozco-Levi M, Guell R, et al. Inspiratory muscle training in patients with chronic obstructive pulmonary disease: structural adaptation and physiologic outcomes. Am J Respir Crit Care Med. 2002; 166(11): 1491-1497, doi 10.1164/rccm.200202-075OC, indexed in Pubmed: 12406842.
12. Severin R, Arena R, Lavie CJ, et al. Respiratory muscle performance screening for infectious disease management following COVID-19: a highly pressurized situation. Am J Med. 2020; 133(9): 1025-1032, doi: 10.1016/j.amjmed.2020.04.003, indexed in Pubmed: 32343947.

13. Bissett B, Gosselink R, Haren Fv. Respiratory Muscle Rehabilitation in Patients with Prolonged Mechanical Ventilation: A Targeted Approach. Annual Update in Intensive Care and Emergency Medicine. 2020: 595-609, doi: 10.1007/978-3-030-37323-8_45. 